\title{
M2 receptors activation modulates cell growth, migration and differentiation of rat Schwann-like adipose-derived stem cells
}

\author{
Roberta Piovesana ${ }^{1,2}$, Alessandro Faroni $\mathbb{C}^{2}$, Valerio Magnaghi ${ }^{3}$, Adam J. Reid ${ }^{2,4}$ and Ada Maria Tata ${ }^{1,5}$
}

\begin{abstract}
Schwann cells (SCs) play a central role in peripheral nervous system physiology and in the response to axon injury. The ability of SCs to proliferate, secrete growth factors, modulate immune response, migrate and re-myelinate regenerating axons has been largely documented. However, there are several restrictions hindering their clinical application, such as the difficulty in collection and a slow in vitro expansion. Adipose-derived stem cells (ASCs) present good properties for peripheral nerve regenerative medicine. When exposed to specific growth factors in vitro, they can acquire a SC-like phenotype (dASCs) expressing key SCs markers and assuming spindle-shaped morphology. Nevertheless, the differentiated phenotype is unstable and several strategies, including pharmacological stimulation, are being studied to improve differentiation outcomes. Cholinergic receptors are potential pharmacological targets expressed in glial cells. Our previous work demonstrated that muscarinic cholinergic receptors, in particular M2 subtype, are present in SCs and are able to modulate several physiological processes. In the present work, muscarinic receptors expression was characterised and the effects mediated by $\mathrm{M} 2$ muscarinic receptor were evaluated in rat dASCS. M2 receptor activation, by the preferred agonist arecaidine propargyl ester (APE), caused a reversible arrest of dASCs cell growth, supported by the downregulation of proteins involved in the maintenance of cell proliferation and upregulation of proteins involved in the differentiation (i.e., c-Jun and Egr-2), without affecting cell survival. Moreover, M2 receptor activation in dASCs enhances a pronounced spindle-shaped morphology, supported by Egr2 upregulation, and inhibits cell migration. Our data clearly demonstrate that rat dASCs express functional muscarinic receptors, in particular M2 subtype, which is able to modulate their physiological and morphological processes, as well as SCs differentiation. These novel findings could open new opportunities for the development of combined cell and pharmacological therapies for peripheral nerve regeneration, harnessing the potential of $\mathrm{dASC}$ and $\mathrm{M} 2$ receptors.
\end{abstract}

Correspondence: Adam J. Reid (Adam.Reid@manchester.ac.uk) or Ada Maria Tata (adamaria.tata@uniroma1.it)

'Department of Biology and Biotechnologies "Charles Darwin", "Sapienza" University of Rome, Rome 00185, Italy

${ }^{2}$ Blond Mclndoe Laboratories, Division of Cell Matrix Biology and Regenerative Medicine, School of Biological Sciences, Faculty of Biology, Medicine and Health, The University of Manchester, Manchester Academic Health Science Centre, Manchester M13 9PT, UK

Full list of author information is available at the end of the article.

Edited by R. Killick

\begin{abstract}
Introduction
Peripheral nerve injuries (PNIs) derived from mechanical trauma or surgical resection secondary to tumour excision are frequent in human. Although the peripheral nervous system (PNS) has a good regeneration capability, several factors can influence nerve repair such as the type and location of injury. However, nerve regeneration outcomes are rarely satisfactory and full functional recovery is often limited ${ }^{1}$. Following injury, neurons and Schwann cells (SCs) change their cellular and molecular properties in order to create a pro-regenerative environment ${ }^{2}$.
\end{abstract}

\section{(c) 2019 The Author(s).}

(c) (i) Open Access This article is licensed under a Creative Commons Attribution 4.0 International License, which permits use, sharing, adaptation, distribution and reproduction cc) in any medium or format, as long as you give appropriate credit to the original author(s) and the source, provide a link to the Creative Commons license, and indicate if changes were made. The images or other third party material in this article are included in the article's Creative Commons license, unless indicated otherwise in a credit line to the material. If material is not included in the article's Creative Commons license and your intended use is not permitted by statutory regulation or exceeds the permitted use, you will need to obtain permission directly from the copyright holder. To view a copy of this license, visit http://creativecommons.org/licenses/by/4.0/. 
Several studies reported that SCs played a relevant role in peripheral regeneration. Regeneration of injured nerves depends on SCs response to produce growth factors, which convert their phenotype into a c-Jun-mediated phenotype called repair $\mathrm{SCs}^{3}$. SCs support nerve regeneration by downregulating myelin genes, upregulating trophic factor production, and increasing cytokine release, all resulting in myelin clearance through a process called myelinophagy, which is mediated by both SCs and macrophages ${ }^{4}$. Finally, SCs form the Büngner's bands which drive regenerating axons towards their targets ${ }^{3}$.

Although SCs role is pivotal after nerve injury, they are not sufficient to support a complete and efficient recovery of peripheral functions clinically. Adipose-derived stem cells (ASCs) may represent an interesting tool for regenerative medicine ${ }^{5,6}$, considering their ability to differentiate into Schwann-like cells (differentiated adiposederived stem cells, dASCs) under appropriate conditions $^{7,8}$. After 18 days of differentiation with selective growth factors, dASCs show properties similar to SCs and they have the ability to improve neurite outgrowth both in vitro and in rodent in vivo models ${ }^{1}$.

Glial cells express receptors for neurotransmitters (i.e., GABA, ATP, acetylcholine), which can modulate cell growth and differentiation ${ }^{9-13}$. Acetylcholine (ACh) involvement in the cross-talk between neurons and glia during neurogenesis, as well as in the adult nervous system, has been widely reported ${ }^{14,15}$.

Previous studies demonstrated that rat SCs express cholinergic muscarinic receptors. In particular, the most abundant receptor subtype is $\mathrm{M} 2^{9,16}$.

Muscarinic acetylcholine receptors are G-protein coupled receptors and, in particular, M2 and M4 receptors are coupled with Gi protein, which causes the inhibition of cyclic AMP (cAMP) production and $\mathrm{K}^{+}$channels modulation ${ }^{14}$.

In SCs, the selective activation of M2 receptors causes a reversible inhibition of cell proliferation, differentiating SCs towards a promyelinating phenotype expressing myelinating transcriptional factors such as Sox10 and Krox20. At the same time the expression of proteins involved in the maintenance of the undifferentiated state (e.g., c-Jun, Notch-1, and Jagged-1) ${ }^{16}$ is suppressed. Myelin alterations and axon degeneration have been also observed in sciatic nerves of M2/M4 KO mice ${ }^{10}$.

dASCs, like SCs, express functional receptors for different neurotransmitters ${ }^{17-19}$. Previously, it has been demonstrated that adipose mesenchymal stem cells (ASCs) are cholinoceptive, expressing different muscarinic receptor subtypes, and in particular, M2 subtype ${ }^{20}$. Recent study shows dASCs express choline acetyltransferase, vesicular acetylcholine transporter and secreting ACh; they promote proliferation of myoblast ${ }^{21}$.
In the present work, we characterised the expression of muscarinic receptors in dASCs and in particular the effects mediated by $\mathrm{M} 2$ receptor activation in terms of cell proliferation and migration. In dASCs, similarly to what observed in SCs, M2 receptor activation caused a reversible decrease of cell proliferation and the inhibition of cell migration. Furthermore, the stimulation of M2 receptors enhanced a pronounced spindle-shaped morphology acquired after growth factor induction, resembling native SCs morphology. Therefore, M2 muscarinic receptor could be a new pharmacological target to regulate important physio-pathological events aimed at improving the recovery from of peripheral nerve injuries.

\section{Results}

Expression of stem cell markers and muscarinic receptors

ASCs express several stem cell markers such as CD29, CD44, CD54 and CD90 ${ }^{20,25,26}$. Firstly, to confirm dASCs differentiation, CD29, CD44 and CD90 expression was evaluated by RT-PCR analysis both in ASCs and dASCs. As expected, after selective growth factors exposure, dASCs showed a significantly downregulated expression of stem cell markers $\left({ }^{* * * *} p<0.001\right.$; Fig. 1a). At the same time dASCs significantly increased the expression of some glial markers such as S100ß, Sox10, GFAP and P0 (Fig. 1b). Moreover, after differentiation, they assumed elongated spindle-shaped morphology, similar to SCs (Fig. 1c) and, as demonstrated by immunostaining, 70\% of the cells express SCs proteins such as GFAP and S100 $\beta$ (Fig. 1d).

Muscarinic receptor expression was characterised in dASCs by RT-PCR analysis, demonstrating that they express the transcripts for $\mathrm{m} 1, \mathrm{~m} 2, \mathrm{~m} 3$ and $\mathrm{m} 4$ subtypes (Fig. 2a). Giving the importance of M2 muscarinic receptor activation in $\mathrm{SCs}^{9,10,16}, \mathrm{M} 2$ muscarinic receptors expression was also investigated by western blot analysis, indicating this receptor subtype is already expressed in ASCs, as previously demonstrated ${ }^{20}$, and its expression is not significantly different in dASCs (Fig. 2b, c).

We also demonstrated that APE $100 \mu \mathrm{M}$ (Arecaidine Propargyl Ester) treatment, activating selectively M2 receptor, induced a significant decrease of cAMP levels in the cells, showing that M2 receptor in dASCs is functional (Fig. 2d). Indeed, APE is able to significantly decrease cAMP levels after $2 \mathrm{~h}$ of treatment, but not following only $30 \mathrm{~min}$ of exposure.

\section{M2 receptor selective activation decreased cell growth}

To investigate the downstream effects mediated by M2 muscarinic receptor activation, cells were treated with APE up to 7 days. APE was used at the final concentration of $100 \mu \mathrm{M}$, the same concentration used previously in SCs and in ASCs experiments ${ }^{10,20}$. 

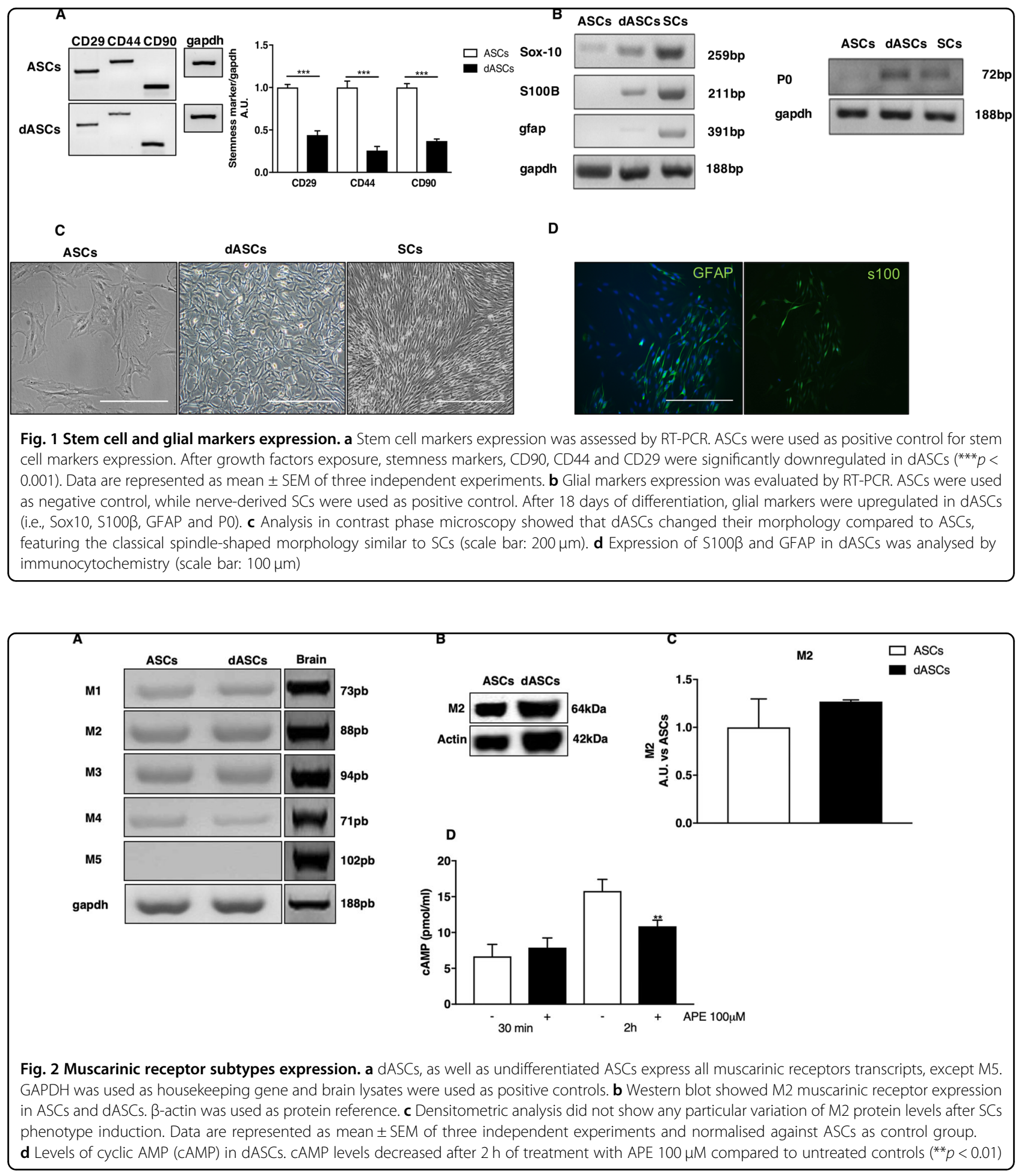

After APE treatment, dASCs cell growth decreased significantly over 7 days in vitro (DIV, Fig. 3a). Moreover, to further confirm that the cell growth inhibition was specifically mediated by $\mathrm{M} 2$ receptor, the M2 antagonist methoctramine was used. The results indicated that $10^{-7} \mathrm{M}$ methoctramine was able to counteract APE effect (Fig. 3a).
Indeed, in the presence of the M2 antagonist, APE was not able to decrease dASCs growth and the number of the cells remained comparable to the untreated cells. The decrease in cell growth was also confirmed by the significant downregulation of genes involved in the control of the cell cycle (i.e., pcna and cyclin D1) (Fig. 3b, c). 


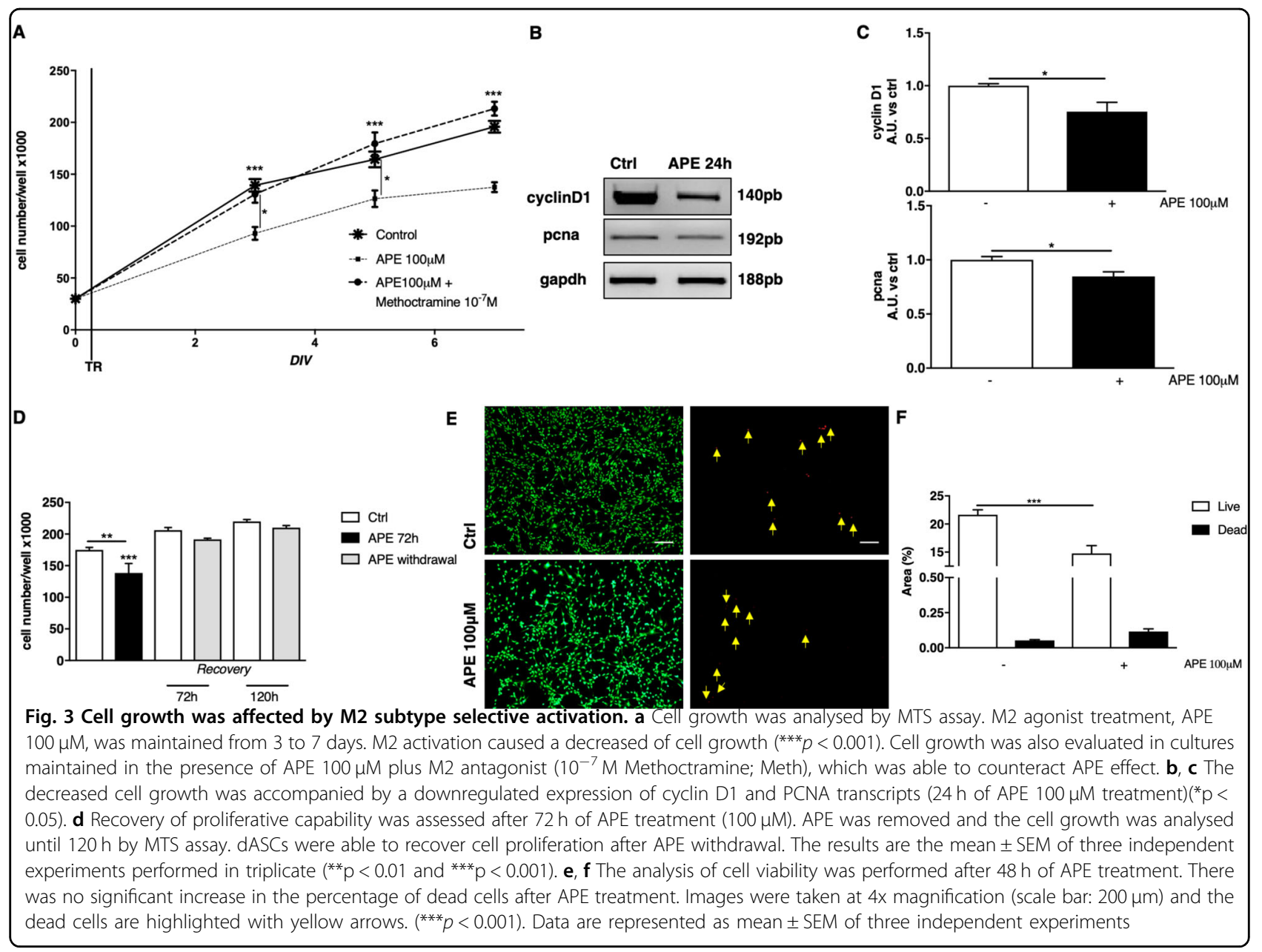

The decreased cell growth was reversible. Indeed, after APE withdrawal, dASCs were able to rescue cell proliferation. In absence of M2 agonist, dASCs were able to recover their ability to proliferate, reaching levels of cell number comparable to untreated controls (Fig. 3d).

In order to exclude the possible toxic effect of APE in dASCs, Live-Dead Assay was performed. The analysis of cell viability confirmed that the total cell number is decreased following APE treatment, as shown by the significant reduction of live cells stain (green, Fig. 3e, f). However, the percentage of dead cells (red) was not significantly modified by the M2 agonist treatment, demonstrating that APE $100 \mu \mathrm{M}$ treatment is not cytotoxic for dASCs (Fig. 3e, f).

\section{M2 receptor activation increased the spindle-shaped morphology and SC-marker expression}

After differentiation, dASCs present a typical spindleshaped morphology $y^{1,7,27}$. APE treatment caused a more evident elongated morphology already after $24 \mathrm{~h}$ of treatment (Fig. 4a, b). After APE treatment, the mean cell diameter of the dASCs decreased and the length increased. The results were graphed as Aspect Ratio, the ratio between the cell length and diameter (Fig. 4b). This ratio significantly increased after $24 \mathrm{~h}$ of APE treatment and progressively increased with the time of treatment. To confirm that aspect ratio increase was dependent on M2 receptor activation, the same experiment was also performed after $72 \mathrm{~h}$ of APE treatment and in presence of M2 antagonist methoctramine $\left(10^{-7} \mathrm{M}\right)$. When M2 antagonist was present, dASCs morphology was similar to the untreated cells and the aspect ratio remained comparable to that of the untreated cells (Fig. 4c). Furthermore, a significant upregulation of myelin protein P0 transcript, a classic SCs marker, corroborates these findings, suggesting improved SCs differentiation (Fig. 4d).

\section{M2 receptor stimulation decreased proliferative related genes and increased egr-2 protein}

In order to validate that the decreased cell number was associated with decreased cell proliferation and possible differentiation, qRT-PCR was used to assess the gene expression changes of some proliferation and differentiation markers. As indicated in Fig. 5a, b, APE 


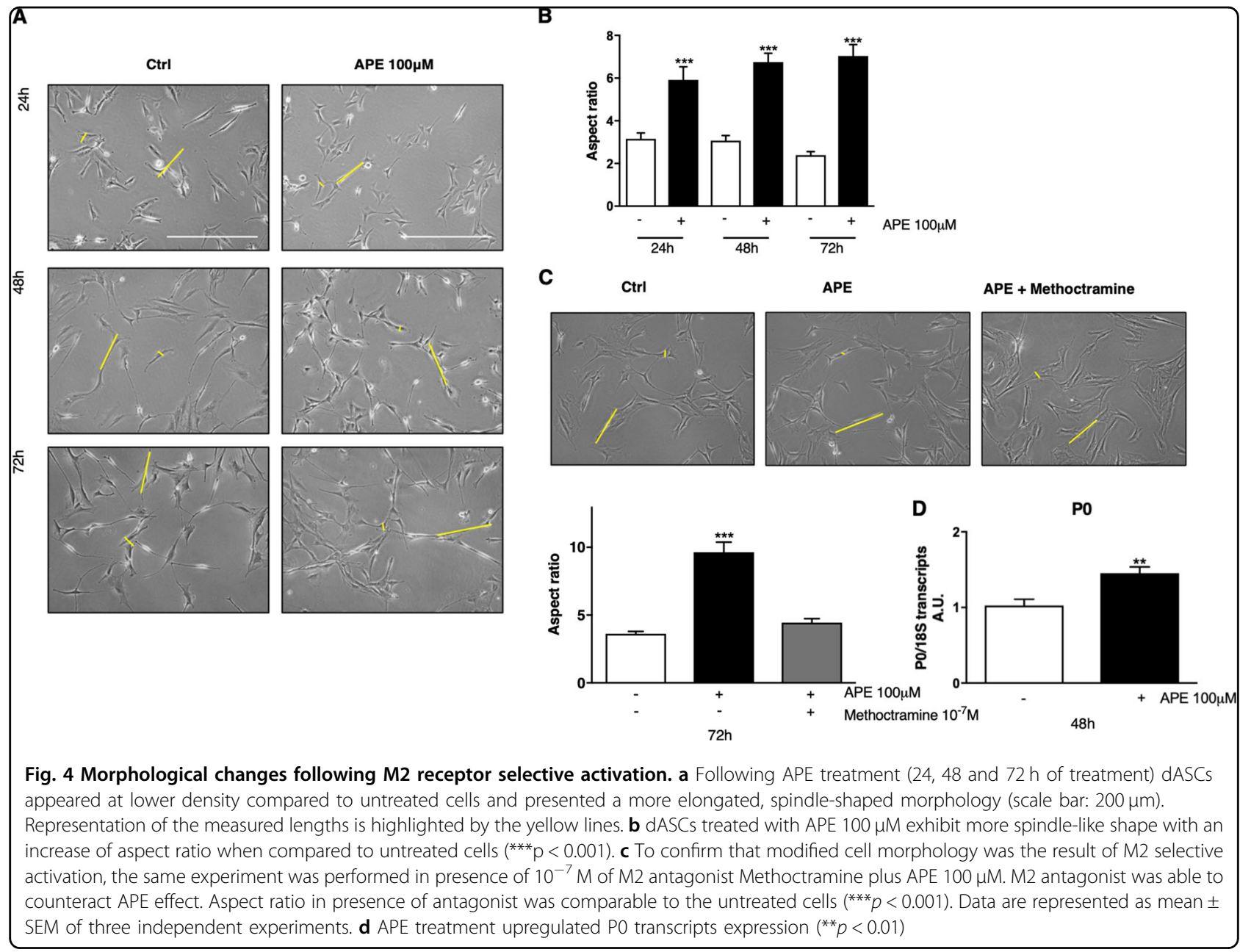

treatment was able to significantly decrease c-jun and notch-1 expression. Interestingly, the decreased expression of c-jun and notch-1 was accompanied by an increased expression of egr-2, a gene associated to SCs differentiation (Fig. 5c). These findings were also confirmed at the protein level. Indeed, M2 selective activation was able to reduce the levels of c-Jun protein significantly after $4 \mathrm{~h}$ and to upregulate Egr-2 protein after $2 \mathrm{~h}$ of treatment (Fig. 5d, e).

Neuregulins (NRG) are proteins expressed by axons and involved in neuron-glia cross talk and some authors described SCs ability to produce NRG-1 in absence of axons $^{28}$. In particular, several isoforms are produced by alternative splicing: NRG1-1 is expressed in a proliferative state, while NRG1-3 isoforms are expressed in myelinating state. By using RT-PCR, we demonstrated that dASCs express NRG1-1 and NRG1-3 isoform transcripts (Fig. 6a). In accordance with MTS results, APE treatment caused a decrease of NRG1-1 transcript expression and an upregulation of NRG1-3, suggesting a switch between a proliferative and a differentiated state (Fig. 6a, b). Moreover, M2 agonist treatment caused an increase of NRG receptor expression, in particular the erbB2 subtype. Conversely, erbB3 receptors transcript levels were not modified by APE treatment (Fig. 6a, b).

\section{M2 receptors selective stimulation altered cell migration}

dASCs migration was analysed after $6 \mathrm{~h}$ by wound healing assay. The results indicated that the presence of M2 agonist APE negatively modulated cell migration. Migration was also measured in presence of methoctramine; M2 antagonist was able to counteract the APE effect and migration was comparable to untreated cells (Fig. 7a, b). After APE treatment, more stress fibres (shown by green Phalloidin staining, Fig. 7a) were present in treated dASCs compared to controls, supporting the decreased migration.

The CXCL12-CXCR7/4 pathway is usually implicated in the control of cell migration and proliferation. In order to explain the M2-mediated mechanisms responsible of cell migration arrest, we analysed the mRNA levels of SDF1/CXCL12 and its receptors CXCR4/CXCR7 by qRTPCR analysis. The results demonstrated that M2 agonist treatment caused a significant downregulation of the 

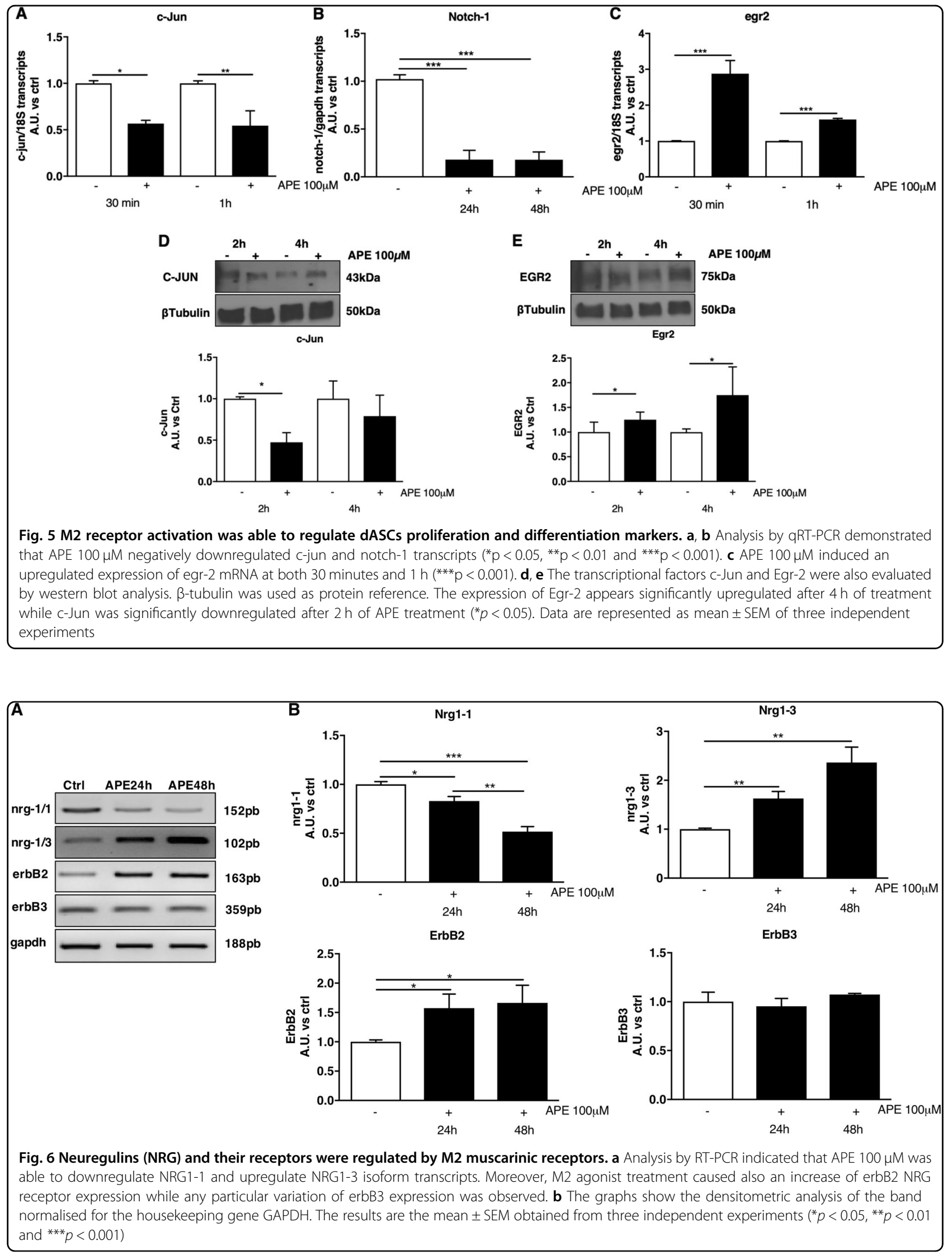


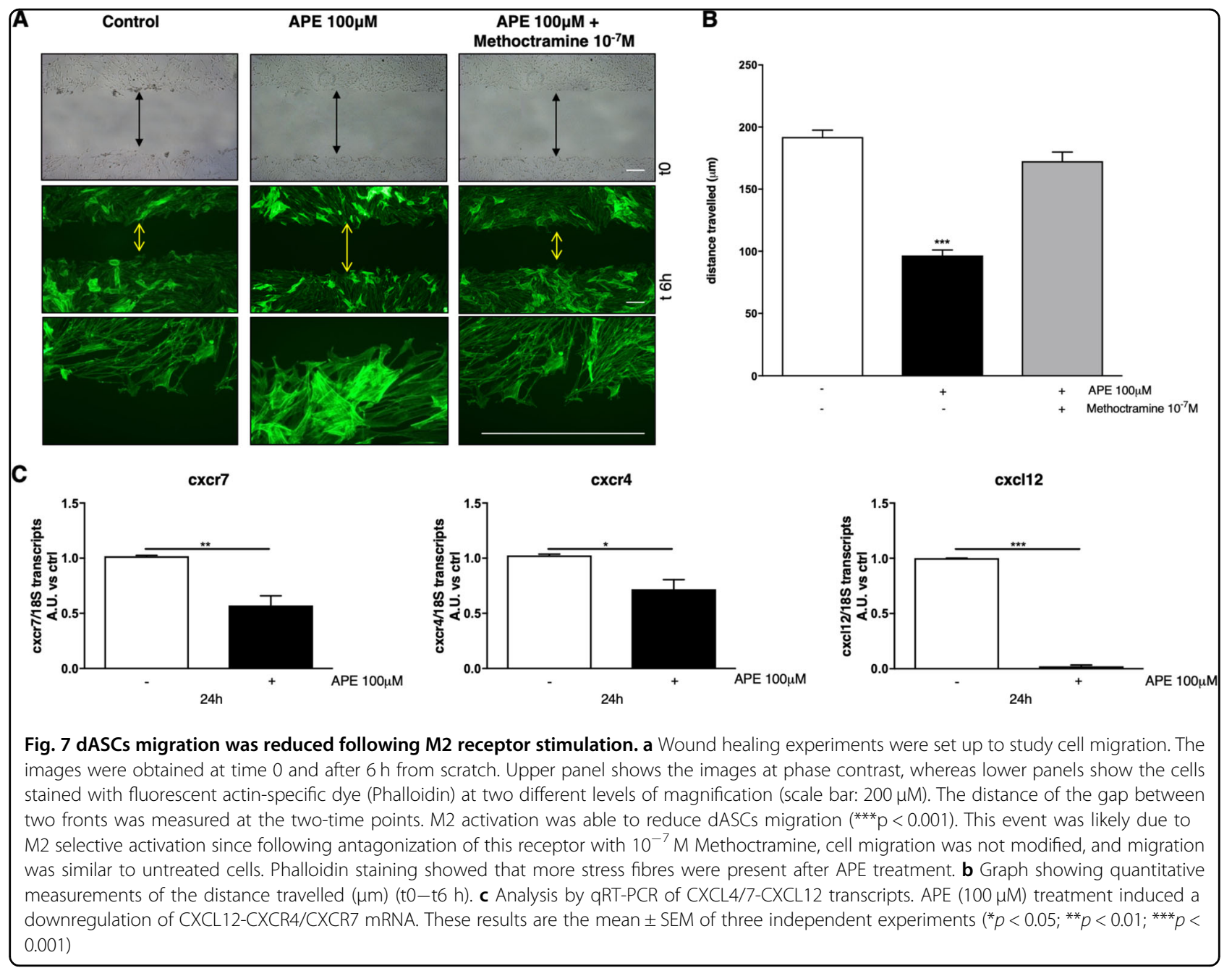

expression of both chemokine transcripts and its receptors after $24 \mathrm{~h}$ of treatment (Fig. 7c).

\section{Discussion}

dASCs derived from ASCs differentiation represent a promising intervention to support and guide peripheral nerve regeneration.

ACh controls neurite outgrowth and, via M2 muscarinic receptors, SCs development ${ }^{10,16}$.

The data reported in the present work clearly demonstrate that dASCs are cholinoceptive and that M2 receptor activation in dASCs produced similar effects to that observed in SCs. The reversible inhibition of cell proliferation, the improved spindle-shaped morphology and the inhibition of cell migration, together with the upregulated expression of SCs differentiation markers such as Egr-2, suggest that the M2 receptor stimulation may strengthen the dASCs spindle-like phenotype and promote differentiation to a SC-like phenotype.
Although the PNS has regenerative capacity, conventional treatments including surgical repair of the damaged nerves, do not achieve complete functional recovery.

The development of novel strategies to enhance peripheral nerve regeneration is, therefore, of great relevance ${ }^{1}$.

Peripheral nerve regeneration is dependent on the greater permissive environment which is likely provided in part by SCs. These cells have a repertoire of physiological roles, including protection and regulation of nutritive exchanges with axons, myelin production and digestion of axons and myelin debris, under pathological conditions. Moreover, SCs promote peripheral nerve regeneration due to their ability to proliferate, migrate and release growth factors driving axon elongation $^{29,30}$. Despite the relevant roles played by SCs during peripheral nerve regeneration, their use in clinical applications is rather limited. Although dASCs may represent an excellent alternative to SCs, they 
quickly revert their phenotype when chemical stimulation is withdrawn ${ }^{27}$.

Considering the effects mediated by M2 muscarinic receptor activation in $\mathrm{SCs}^{10,16}$ and in $\mathrm{ASCs}^{20}$, it seems a relevant tool to characterise $\mathrm{M} 2$ receptors activation also in dASCs. These cells combined with novel treatments may represent a successful strategy in the clinical treatment of peripheral nerve lesions ${ }^{31}$.

Unlike other neurotransmitter receptors, in this manuscript, we showed that M2 receptor expression did not change following dASCs differentiation compared to ASCs. It is known that M2 receptor is able to reduce cAMP concentration via Gi-protein ${ }^{32}$. Our previous studies have clearly demonstrated by pharmacological experiments and M2 knockdown that APE selectively binds only M2 receptors ${ }^{10,33}$. APE treatment was able to reduce cAMP concentration, supporting the functional presence of M2 receptors on dASCs membrane.

Similar to observations in SCs, the pharmacological activation of M2 muscarinic receptors reduced cell growth, without influencing cell viability. This was demonstrated by proliferation and live-dead studies, and corroborated by the downregulation of genes involved in the control of cell cycle (i.e., pcna and cyclinD1).

The inhibitory effect of M2 agonist on cell proliferation was however reversible; APE withdrawal from culture medium caused a rescue of dASCs proliferation, confirming that M2 activation maintained cell plasticity, as observed in SCs and $\mathrm{ASCs}^{16,20}$.

SCs morphology depends on the physiological state of the cells; they change their morphology in order to participate at different physiological and pathological events $^{34}$. The chemical differentiation via growth factors changes ASCs morphology, decreasing the cell diameter and improving cell length, in a phenotype that resembles native SCs.

Interestingly, M2 receptor activation caused a significant change in dASCs cell morphology, stimulating a more evident spindle-like phenotype. It is possible to speculate that these changes are a sign of occuring differentiation events. In this manuscript, we showed that dASCs expressed NRG1 isoforms transcript. NRG1 is an axonal factor, that supports nerve regeneration ${ }^{35}$; SCs are also able to produce this factor in autocrine way supporting survival and proliferation also in the absence of axons ${ }^{28}$. Indeed, several studies showed that SCs recapitulate a nerve injury condition in vitro ${ }^{36}$. Moreover, NRG1 is able to control many aspects of SCs differentiation and myelination ${ }^{37}$. It has recently been shown that withdrawal of NRG1-1 in dASCs decreased the expression of SCs mar$\mathrm{kers}^{38}$, and it is able to improve SCs-like phenotype in association with other growth factors (e.g., FGF, PDGF and Fsk $)^{39}$. We showed that M2 stimulation upregulated NRG1-3, the isoform involved in the myelinating phenotype, suggesting an improvement in the SC-like phenotype. Our studies clearly demonstrated an upregulation of NRG, followed by an early upregulation of Egr2/ Krox20 expression. We have previously shown that NRG alone can increase Egr2/Krox20 expression without M2 stimulation ${ }^{39}$, nevertheless, in this study we show that the synergistic action of NRG and M2 agonist further increases Egr2/Krox20 expression.

There is much evidence to indicate that SCs differentiation in myelinating phenotype depends on Krox-20/ Egr2 and other promyelin transcription factors, that are normally activated by axonal signals ${ }^{40}$. Moreover, c-Jun is an important regulator of SCs plasticity and it is able to inhibit myelin related genes ${ }^{3}$. It is interesting that c-Jun and Egr-2 show a cross-antagonistic functional relationship. In our studies, we demonstrated that M2 selective activation was able to downregulate c-Jun after $2 \mathrm{~h}$ of treatment. Interestingly, this downregulation was accompanied by an upregulation of Egr-2, as described in the literature. This result supports the hypothesis that these cells respond in a similar way to SCs under physiological conditions; moreover, it is shown that $\mathrm{ACh}$, via M2 receptor, is able to regulate their physiology, as indicated by morphological changes related to Egr-2 upregulation.

Another important event in the injury response is SCs migration. SCs are able to proliferate and then migrate to the injured site to help environmental clearance and nerve regeneration ${ }^{41}$. Other authors described that ACh stimulation in the presence of calcium enhanced migratory capacity via M1 subtypes in mesenchymal stem cells ${ }^{42}$. Herein, we showed that APE treatment, via M2 receptor stimulation, caused a decrease of cell migration in dASCs. In particular, after APE treatment, cells reorganised their cytoskeleton, showed a decreased cell migration, accompanied by a downregulated expression of CXCL12/SDF-1 and its receptors CXCR7 and CXCR4. This pathway is involved in the control of SCs migration during regeneration $^{43}$ and dASCs also express and secrete chemokines and their receptors that modulate their migration activity ${ }^{44}$. In accordance with the observed inhibitory effect of APE on dASCs migration, the expression levels of CXCL12/SDF-1 and its receptors CXCR7 and CXCR4 were downregulated.

Albeit the ability of APE to selectively activate M2 receptor subtype has been largely demonstrated in our previous works ${ }^{15,16,20,33}$, to confirm APE specificity for M2 receptor, we performed co-treatments with the specific M2 antagonist methoctramine. The results obtained for the analyses of cell growth and cell migration, as well as the study of dASCs morphology, demonstrated that the presence of M2 antagonist counteracted the APE effects.

Altogether, these findings agree with reports in other papers and raise the possibility of other neurotransmitter 
receptors (i.e. GABA and purinergic receptors) to modulate ASCs and dASCs physiological processes ${ }^{18,19,39}$. In this scenario, neurotransmitter receptors could be potential pharmacological targets to modulate ASCs physiology and differentiation towards dASCs phenotype.

Although further analyses are needed to fully understand the role of M2 receptor in differentiation and the role of the other muscarinic receptor subtypes in dASCs, our data suggest that the activation of muscarinic receptors influences the physiology of the dASCs and could potentially represent a therapeutic target for nerve regeneration. In this context, we believe that these findings could lead to a novel strategy for nerve repair, combining the potential of stem cell therapy together with the pharmacological stimulation of neurotransmitters receptors on transplanted ASCs.

\section{Materials}

\section{Adipose-derived stem cells harvesting and culture}

The experimental procedures that involved animals were performed in accordance with the Animals (scientific procedures) Act 1986. ASCs were isolated from adult Sprague-Dawley rats as described previously ${ }^{7}$. Fat pads were dissected and chopped using a sterile razor blade. After, tissue was enzymatically digested for $1 \mathrm{~h}$ at $37^{\circ} \mathrm{C}$ using $0.15 \%(\mathrm{w} / \mathrm{v})$ collagenase type I (Invitrogen, UK). A $100 \mu \mathrm{m}$ filter was used to remove the undissociated tissue. The solution was centrifugated at $1200 \mathrm{rpm}$ for $10 \mathrm{~min}$, and the stromal vascular fraction (SVF) was obtained. The resultant SVF includes preadipocytes, endothelial cells, macrophages, fibroblasts and adipose-derived stem cells (ASCs), which are able to adhere to plastic and proliferate rapidly than the other cell type ${ }^{22}$. The stromal cell pellet was plated in $75 \mathrm{~cm}^{2}$ cell culture flasks in stem cell growth medium consisting in alpha minimum essential medium $(\alpha$-MEM) supplemented with $10 \%(v / v)$ fetal bovine serum (FBS), 2mM L-glutamine and 1\% (v/v) Penicillin/ Streptomycin solution. Cultures were maintained at subconfluent levels in a $37^{\circ} \mathrm{C}$ incubator with $5 \% \mathrm{CO}_{2}$.

\section{Stem cells differentiation to Schwann-like phenotype}

For differentiation to SCs phenotype, at passage 1-2 ASCs were treated with stem cell growth medium supplemented with $1 \mathrm{mM} \beta$-mercaptoethanol for $24 \mathrm{~h}$. The next day, cells were incubated with $10 \mathrm{ml}$ of preconditioning medium for $72 \mathrm{~h}$ containing $35 \mathrm{ng} / \mathrm{ml}$ alltrans-retinoic acid at $37^{\circ} \mathrm{C}$. Following all-trans-retinoic acid treatment, cells were washed carefully and stem cell medium was replaced supplemented with $14 \mu \mathrm{M}$ forskolin, $192 \mathrm{ng} / \mathrm{ml}$ glial growth factor-2 (GGF-2, Acorda, $\mathrm{UK}$ ), $5 \mathrm{ng} / \mathrm{mL}$ platelet-derived growth factor (PDGF, Peprotech, USA), and $10 \mathrm{ng} / \mathrm{ml}$ basic fibroblast growth factor (bFGF Peprotech, USA) for 14 days $^{23}$. The same supplemented medium was used for cell maintenance.
Cells were incubated at humidified $37^{\circ} \mathrm{C}$ environment with $5 \% \mathrm{CO}_{2}$. SCs cultures were obtained from sciatic nerves of P1-P2 Sprague-Dawley rats using a previously established protocol ${ }^{17,24}$ and used as positive controls for SC-like differentiation.

\section{Experimental set up and pharmacological treatments}

M2 muscarinic receptor agonist, Arecaidine propargyl ester hydrobromide (APE, Sigma-Aldrich, St. Louis, MO, USA) was used at the final concentration of $100 \mu \mathrm{M}$, according to previous studies ${ }^{10,20}$. M2 muscarinic receptor antagonist, methoctramine, was used at final concentration of $10^{-7} \mathrm{M}$ (Meth, Sigma-Aldrich, St. Louis, MO, USA). M2 muscarinic receptor antagonist was added $2 \mathrm{~h}$ before APE treatment. Controls were obtained maintaining the cells in normal growth medium. Technical and experimental triplicates were performed for all experiments.

\section{RT-PCR and quantitative real-time PCR (qPCR)}

Cells were collected at the time point chosen and stored in RNA cell protect agent (Qiagen, Manchester, UK). Total RNA was isolated from dASCs using RNeasy Plus Mini Kit (Qiagen, Manchester, UK), according to the manufacturer's protocol. Each sample was reverse-trascripted using $\mathrm{RT}^{2}$ First Strand Kit (Qiagen, Manchester, UK), according to the manufacturer's protocol. cDNA was used in RT-PCR and primers and GoTaq Green Master Mix (Promega, Madison, WI, USA) were added. For semiquantitative RT-PCR the densitometric analysis of the bands were performed using ImageJ software (NIH, Bethesda, MA, USA) (OD amplicon/ OD housekeeping gene). These values are expressed as arbitrary units. Quantitative real-time PCR was performed with $\mathrm{RT}^{2}$ SYBR Green qPCR Mastermix (Qiagen, Manchester, UK) using Corbett Rotor Gene 6000 real-time cycler (Qiagen, UK). All reactions were carried out in triplicate and the protocol used was: hot start for $10 \mathrm{~min}$ at $95^{\circ} \mathrm{C}$, followed by 45 cycles of $15 \mathrm{~s}$ at $95^{\circ} \mathrm{C}$, annealing for $30 \mathrm{~s}$ at $55^{\circ} \mathrm{C}$ and extension for $30 \mathrm{~s}$ at $72^{\circ} \mathrm{C}$. The sequences of the primers used are reported in Table 1. Data were normalised with housekeeping gene (18S or gapdh) and the $\Delta \Delta \mathrm{Ct}$ method was used to determine the fold changes in the gene expression, as compared to control.

\section{Western blot}

Whole-cells lysates were obtained by scraping cells from confluent 6 well-plates using RIPA Buffer (Sigma-Aldrich, UK) supplemented with a cocktail of protease and phophatase inhibitors (Thermo Scientific, Loughborough, UK). Lysates were incubated for $30 \mathrm{~min}$ on ice and then centrifuged for $20 \mathrm{~min}$ at $14 \mathrm{rpm}$ at $4{ }^{\circ} \mathrm{C}$. Protein concentration was determined using Pierce ${ }^{\mathrm{mw}}$ BCA Protein Assay Kit (Thermo Scientific, Waltham, MA, USA), according to the manufacturer's protocol. Sample buffer $(6 \times)$ was added to protein lysates and heated for $5 \mathrm{~min}$ at 
Table 1 Primer sequences used in semiquantitative and quantitative RT-PCR analysis

\begin{tabular}{|c|c|c|}
\hline Gene & Forward & Reverse \\
\hline mAChR M1 & 5'-CCATGGAGTCCCTCACATCCT-3' & 5'-ATCTACCATGGGCATCTTGATCA-3' \\
\hline mAChR M2 & 5'-GCTCCAATGATTCGACGTCA-3' & 5'-CGAAGTGGAAACTGTTGTITTCAT-3' \\
\hline mAChR M3 & 5'-TCCATCCTCAACTCTACCAAGCT-3' & 5'-TTGTGAGCATTTCTCTCCACATC-3' \\
\hline mAChR M4 & 5'-CACTCTGCAATGCCACTTTCAA-3' & 5'-CTGTGCCGATGTTCCGATACT-3' \\
\hline mAChR M5 & 5'-ACCCGCACTGAAAACAGTGACT-3' & 5'-ATCGGAACTAGGCAACACACTT-3' \\
\hline CD29 & 5'-TGGGTCGCTGATTGGCTG-3' & 5'-CTCTTCAGTGACTGCAAAAATCG-3' \\
\hline CD44 & 5'-TCATGTTAGAGCATCCGTGC-3' & 5'-GGGTTGTACATCATGCCTCC-3' \\
\hline CD90 & 5'-TGAACCCAGTCATCAGCAT-3' & 5'-CAGTCGAAGGTTCTGGTTCACC-3' \\
\hline cyclinD1 & 5'-CCCTCTGCACGCACTTGAAG-3' & 5'-GCGAGCCATGCTTAAGACTGA-3' \\
\hline pena & 5'-GAAGCACCAAATCAAGAGAA-3' & 5'-TCACCCCGTCCTITGCACAG-3' \\
\hline notch1 & 5'-CTCACGCTGATGTCAATGCT-3' & 5'-GCAACACTTTGGCAGTCTTCA-3' \\
\hline$c-j u n$ & 5'-GATGGAAACGACCTTCTACGAC-3' & 5'-AGCGTATTCTGGCTATGCAGTT-3' \\
\hline $50 \times 10$ & 5'-ACTGGGAACAGCCAGTATATA-3' & 5'-ACCAAACTCCTCCTTTGCCA-3' \\
\hline$S 100 B$ & 5'-ATAGCACCTCCGTTGGACAG-3' & 5'-TCGTTTGCACAGAGGACAAG-3' \\
\hline gfap & 5'-GCTGAACTGAACCAGCTTCGA-3' & 5'-CTTGGCCACATCCATCTCCAC-3' \\
\hline po & 5'-TCTITACCTGGCGCTACCAG-3' & 5'-GTTGACCCTTGGCATAGTGGA-3' \\
\hline egr2 & 5'-AACGGAGTGGCCGGAGAT-3' & 5'-ATGGGAGATCCAACGACCTCTT-3' \\
\hline$n r g / / 1$ & 5'-TCATCTTCGGCGAGATGTCTG-3' & 5-'CTCCTGGCTTTCATTTCTITCA-3' \\
\hline$n r g / / I I$ & 5'-GGACCCCTGAGGTGAGAGAACA-3' & 5'-CAGTCGTGGATGTCGATGTGG-3' \\
\hline erbB2 & 5'-CGAGTGTCAGCCTCAAAACA-3' & 5'-CTCATCCGGGTACTTCCAGA-3' \\
\hline erbB3 & 5'-CTGTTTAGGCCAAGCAGAGG-3' & 5'-GACTTTGTTTGCCTTCTCGC-3' \\
\hline CXCR4 & 5'-GCCATGGCTGACTGGTACTT-3' & 5'-GATGAAGGCCAGGATGAGAA-3' \\
\hline CXCR7 & 5'-GGCTACGACACACACTGCTA-3' & 5'-GGTCCACGCTCATGCATGCG-3' \\
\hline CXCL12 & 5'-TGCATCAGTGACGGTAAGCCA-3' & 5'-ATCCACTTTAATTTCGGGTCAA-3' \\
\hline $18 \mathrm{~S}$ & 5'-GGATCCATTGGAGGGCAAGT-3' & 5'-ACGAGCTIIITAACTGCAGCAA-3' \\
\hline Gapdh & 5'-GTGCCAGCCTCGTCTCATAG-3' & 5'-TGATGGCAACAATGTCCACT-3' \\
\hline
\end{tabular}

$100{ }^{\circ} \mathrm{C}$. Afterwards, $30 \mu \mathrm{g}$ of the sample was loaded onto a $10 \%$ SDS (Sodium dodecyl sulfate) polyacrylamide gel and run at $120 \mathrm{~V}$ using running buffer $(25 \mathrm{mM}$ Tris, $190 \mathrm{mM}$ glycine, $0,08 \%(\mathrm{w} / \mathrm{v})$ SDS). SDS-PAGE gels were transferred for $1 \mathrm{~h}$ onto nitrocellulose blotting membranes (GE Healthcare Life Science, Amersham, Germany) at $80 \mathrm{~V}$ in transfer buffer (25 mM Tris-base; $192 \mathrm{nM}$ glycine, 20\% (v/v) methanol). After transfer, membranes were blocked for $1 \mathrm{~h}$ in a Tris-buffer saline (TBS)- Tween Solution containing $5 \%$ non-fat dry milk. Membranes were incubated with the primary antibody, diluted in blocking buffer, overnight at $4{ }^{\circ} \mathrm{C}$. Primary antibodies used were: Mouse anti-M2 antibody (1:500, Abcam, UK), rabbit antic-Jun (1:1000, Abcam, UK), rabbit anti-Egr-2 (1:500, ProteinTech, UK). After overnight incubation, membranes were washed with TBS-Tween buffer and thus incubated for $1 \mathrm{~h}$ at room temperature with secondary antibody: anti-rabbit horseradish peroxidase (1:2000, Cell Signaling, Hitchin, UK) or anti-mouse horseradish peroxidase (1:1000, Cell Signaling, Hitchin, UK) for chemiluminescence detection. Actin or $\beta$-tubulin was used as protein reference (mouse anti-actin, 1:6000, (Millipore), rabbit anti- $\beta$-tubulin (1:1000, Abcam, Cambridge, UK)). To determine housekeeping protein, membranes were stripped before re-blotting with another primary antibody. Membranes were exposed to SuperSignal West Pico Chemiluminescent Substrate (Thermo Scientific, Waltham, MA, USA) for signal detection.

\section{Immunocytochemistry}

Cells were seeded into $35 \mathrm{~mm}^{2}$ plates in complete medium at the density of $2 \times 10^{4} / \mathrm{ml}$. 
At subconfluence, cells were washed twice with PBS and fixed with 4\% PFA (paraformaldehyde, PFA) for $20 \mathrm{~min}$ at room temperature (RT). Cells were incubated with Triton-X $0,2 \%$ for $30 \mathrm{~min}$ at RT. Then cells were washed twice with PBS and treated with block solution (PBS 0.1\% Triton X-100 and 10\% normal donkey serum (NGS) for $1 \mathrm{~h}$ at RT. The cells were incubated with antirabbit-GFAP (1:100, DAKO, Glostrup, Denmark) and anti-rabbit-S100 $\beta$ (1:100, DAKO, Glostrup, Denmark) at $4{ }^{\circ} \mathrm{C}$ overnight. The day after, cells were washed with PBS three times for $10 \mathrm{~min}$ and they were incubated with goat anti-rabbit IgG Alexa Flour 488-conjugated antibody (Life Technologies, UK), diluted 1:500 in 0.1\% Triton X-100, 0,1\% (w/v) BSA, 0,1\% (w/v) Sodium Azide in PBS for $1 \mathrm{~h}$ at RT. After, plates were washed 3 times with PBS and slides were mounted with Vectashield mounting medium for fluorescence containing 4'-6'diamidino-2-phenylindole for nuclear staining ( $\mathrm{H} 1200$, Vector Lab, DBA, Milan, Italy). Images were taken using a fluorescence microscope (Olympus IX51, Sounthendon-Sea, UK) and processed with ImageJ 64 imaging software (National Institutes of Health, NIH, Bethesda, MD, USA).

\section{Measurement of cAMP levels}

Cells were seeded on 6-well plate at the density of $25 \times 10^{3}$ cells/well. When cells were at $80 \%$ confluent, they were treated with APE $100 \mu \mathrm{M}$. Each condition was done in triplicate and samples from the same groups were pooled. cAMP analyses were performed using cyclic AMP Select ELISA kit (Cayman Chemical, MI, USA), according to the manufacturer's protocol. Plates were read with an Asys UVM-340 microplate reader/spectrophotometer (Biochrom, Cambridge, UK). Absorbance was recorded at $420 \mathrm{~nm}$.

\section{Proliferation assay}

To study cell proliferation, dASCs were seeded on 24-well plates at the density of $25 \times 10^{3}$ cells/well. After $24 \mathrm{~h}$, cells were treated with APE $100 \mu \mathrm{M}$ (Sigma-Aldrich, St. Louis, MO, USA) for 3, 5, 7 days. M2 muscarinic receptor antagonist methoctramine $\left(10^{-7} \mathrm{M}\right.$, Sigma-Aldrich, St. Louis, MO, USA) was used $2 \mathrm{~h}$ before APE treatment, when required. This method is based on a tetrazolium compound reduction generating a colored formazan product by viable cells; this is soluble in cell culture media. Cells were incubated in 20\% (v/v) CellTiter 96 AQueous One Solution Cell Proliferation Assay (Promega, Southampton, UK), diluited in phenol-free DMEM (Sigma-Aldrich, UK) for $90 \mathrm{~min}$ at $37^{\circ} \mathrm{C}$ in the dark. For each well, the absorbance at $490 \mathrm{~nm}$ was recorded with an Asys UVM-340 microplate reader/ spectrophotometer (Biochrom, Cambridge, UK). After a standard curve was performed, data were expressed as cell number \pm SEM of the mean.

\section{Live dead assay}

Cell viability was analysed by using LIVE/DEAD Viability/ Cytotoxicity Kit for mammalian cells (Molecular Probes, Invitrogen, UK). Calcein-AM was used to identify live cells (green fluorescence; $\lambda=498 \mathrm{~nm}$ ), conversely Eth-D1 was used to identify dead cells (red fluorescence; $\lambda=635 \mathrm{~nm}$ ). dASCs were plated in a concentration of $40 \times 10^{3}$ cells/well into 12 well plates. Calcein-AM and Eth-D1 were added at a concentration of $0.5 \mu \mathrm{l} / \mathrm{ml}$ and $2 \mu \mathrm{l} / \mathrm{ml}$, respectively, and cells were incubated for $30 \mathrm{~min}$ at RT, and then washed in PBS prior to imaging. Images were taken using fluorescence inverted microscope (Olympus IX51, Sounthend-on-Sea, $\mathrm{UK})$ and processed with Image 64 imaging software $(\mathrm{NIH}$, Bethesda, MD, USA) under 4x magnification.

\section{Recovery assay}

Recovery analysis was set up to demonstrate whether inhibition of proliferation-APE induced was reversible. The cells were treated for $72 \mathrm{~h}$ with APE. Then, complete medium containing APE was removed; the cells were washed once with sterile PBS, and fresh complete media added. Growth analysis was assessed as described above. For each well, the absorbance at $490 \mathrm{~nm}$ was recorded with an Asys UVM-340 microplate reader/spectrophotometer (Biochrom, Cambridge, UK). Stardard curve was performed and the data were expressed as cell number \pm SEM of the mean.

\section{Wound healing}

Cell migration was evaluated into six-well plates in triplicate for all conditions. Wound was generated with p200 tip and, to exclude possible proliferation interference, Mitomycin $\mathrm{C}$ was also added to the cell culture media (50 ng/ml, Sigma-Aldrich). The cells were photographed at time 0 and after $6 \mathrm{~h}$ at $4 \mathrm{x}$ magnification. The space was measured between two fronts at time $0 \mathrm{~h}$ (T0) and $6 \mathrm{~h}$ (T6). The two values were subtracted, obtaining the covered space by the cells in the experimental time chosen. After $6 \mathrm{~h}$, cells were fixed with $4 \%$ PFA in PBS (SigmaAldrich, UK), permealized for $15 \mathrm{~min}$ with $0.2 \%(\mathrm{v} / \mathrm{v})$ Triton-X in PBS (Sigma-Aldrich, UK) and stained for 20 min at RT with Alexa 488-conjugated phalloidin (1:40, Life Technologies, UK) diluted in 1\% (w/v) BSA in PBS. Images were taken using a fluorescence microscope at $4 \mathrm{x}$ magnification (Olympus IX51, Sounthend-on-Sea, UK) and processed with ImageJ 64 imaging software $(\mathrm{NIH}$, Bethesda, MD, USA).

\section{Cell morphology assessment}

To determine individual cell morphology dASCs were plated in six-well plates in triplicate at a density of $50 \times$ $10^{3}$ cells/well. After $24 \mathrm{~h}$, cells were treated with APE $100 \mu \mathrm{M}$ (Sigma-Aldrich, St. Louis, MO, USA) or APE plus M2 antagonist methoctramine (Meth $10^{-7}$ M, Sigma- 
Aldrich, St. Louis, MO, USA). The time points chosen were from 1 to 3 days after treatment.

Images were captured with an Olympus IX51 wide-field microscope at 10x magnification. Images were analysed with IMAGE J (v1.47f; NIH, Bethesda, MD, USA) to measure cell length (longest cell length) and width (narrowest cell width). The aspect ratio was calculated as length/width for each cell measured.

\section{Statistical analysis}

Data analysis were performed with GraphPad Prism (ver 7.0, GraphPad Software Inc, La Jolla, CA, USA). Data were presented as the mean \pm SEM. Student's $t$-test or one-way ANOVA analyses with Bonferroni post-tests were used. A value of $p<0.05$ was considered statistically significant. ( $p$ $<0.05{ }^{(*)}, p<0.01{ }^{(* *)}$ and $\left.p<0.001{ }^{(* * *)}\right)$.

\section{Acknowledgements}

The authors are grateful to Prof. Terenghi for critical reading of the manuscript. This work was supported by Ateneo Sapienza Funds to A.M.T. R.P. fellowship was supported by Torno Subito Project 2015 (Lazio, Italy) Avvio Giovani 2018 and CIB'fellowship 2018. A.F. and A.J.R. are supported by the Hargreaves and Ball Trust and the Academy of Medical Sciences. Thank you to Acorda Therapeutics for kindly supplying us with recombinant GGF-2 for use in this study. This work was also supported by a grant from MIUR "Progetto Eccellenza" to V.M.

\section{Author details}

"Department of Biology and Biotechnologies "Charles Darwin", "Sapienza" University of Rome, Rome 00185, Italy. ${ }^{2}$ Blond Mclndoe Laboratories, Division of Cell Matrix Biology and Regenerative Medicine, School of Biological Sciences, Faculty of Biology, Medicine and Health, The University of Manchester, Manchester Academic Health Science Centre, Manchester M13 9PT, UK. ${ }^{3}$ Department of Pharmacological and Biomolecular Sciences, University of Milan, Milan 20133, Italy. ${ }^{4}$ Department of Plastic Surgery \& Burns, Wythenshawe Hospital, Manchester University NHS Foundation Trust, Manchester Academic Health Science Centre, Manchester, UK. ${ }^{5}$ Research Center of Neurobiology "Daniel Bovet", "Sapienza" University of Rome, Rome 00185, Italy

\section{Authors' contributions}

Conception and design: A.M.T., R.P., A.F., V.M. and A.J.R. Acquisition of data: R.P. and A.F. Preparation of dASCs: R.P. and A.F. Analysis and interpretation of data: R.P., A.F., A.M.T., A.J.R. and V.M. Drafting the article: R.P. and A.M.T. Critical revision of this article: A.J.R., A.F. and V.M. The final version of the manuscript was approved by all authors.

\section{Conflict of interest}

The authors declare that they have no conflict of interest.

\section{Publisher's note}

Springer Nature remains neutral with regard to jurisdictional claims in published maps and institutional affiliations.

Received: 4 January 2019 Revised: 13 February 2019 Accepted: 4 March 2019

Published online: 03 May 2019

\section{References}

1. Faroni, A., Mobasseri, S. A., Kingham, P. J. \& Reid, A. J. Peripheral nerve regeneration: experimental strategies and future perspectives. Adv. Drug Deliv. Rev. 82, 160-167 (2015).
2. Dubový, P. Wallerian degeneration and peripheral nerve conditions for both axonal regeneration and neuropathic pain induction. Ann. Anat. 193, 267-275 (2011).

3. Jessen, K. R. \& Mirsky, R. The repair Schwann cell and its function in regenerating nerves. J. Physiol. 594, 3521-3531 (2016).

4. Gomez-Sanchez, J. A. et al. Schwann cell autophagy, myelinophagy, initiates myelin clearance from injured nerves. J. Cell. Biol. 210, 153-168 (2015).

5. Gimble, J. M., Katz, A. J. \& Bunnell, B. A. Adipose-derived stem cells for regenerative medicine. Circ. Res. 100, 1249-1260 (2017).

6. Guo, J., Guo, S., Wang, Y. \& Yu, Y. Promoting potential of adipose derived stem cells on peripheral nerve regeneration. Mol. Med. Rep. 16, 7297-7304 (2017).

7. Kingham, P. J. et al. Adipose-derived stem cells differentiate into a Schwann cell phenotype and promote neurite outgrowth in vitro. Exp. Neurol. 207, 267-274 (2007)

8. Mantovani, C. et al. Bone marrow- and adipose-derived stem cells show expression of myelin mRNAs and proteins. Regen. Med. 5, 403-410 (2010).

9. Loreti, S. et al. Rat Schwann cells express M1-M4 muscarinic receptor subtypes. J. Neurosci. Res. 84, 97-105 (2006).

10. Uggenti, C. et al. M2 muscarinic receptor activation regulates schwann cell differentiation and myelin organization. Dev. Neurobiol. 74, 676-691 (2014).

11. Luo, J. et al. P2X7 purinoceptors contribute to the death of Schwann cells transplanted into the spinal cord. Cell. Death. Dis. 4, 1-10 (2013).

12. Faroni, $A$. et al. Deletion of GABA-B receptor in schwann cells regulates remak bundles and small nociceptive C-fibers. Glia 62, 548-565 (2014).

13. Magnaghi, $\vee$. Nerve regenerative effects of GABA-B ligands in a model of neuropathic pain. Biomed. Res.Int. 2014, 368678 (2014).

14. Fields, R. D., Dutta, D. J., Belgrad, J. \& Robnett, M. Cholinergic signaling in myelination. Glia 65, 687-698 (2017).

15. De Angelis, F., Bernardo, A., Magnaghi, V., Minghetti, L. \& Tata, A. M. Muscarinic receptor subtypes as potential targets to modulate oligodendrocyte progenitor survival, proliferation, and differentiation. Dev. Neurobiol. 72, 713-728 (2012).

16. Loreti, S., Ricordy, R., De Stefano, M. E., Augusti-Tocco, G. \& Tata, A. M. Acetylcholine inhibits cell cycle progression in rat Schwann cells by activation of the M2 receptor subtype. Neuron Glia Biol. 3, 269-279 (2007).

17. Faroni, A. et al. Schwann-like adult stem cells derived from bone marrow and adipose tissue express $\gamma$-aminobutyric acid type B receptors. J. Neurosci. Res. 89, 1351-1362 (2011).

18. Faroni, A., Terenghi, G. \& Magnaghi, V. Expression of functional $\gamma$-aminobutyric acid type A receptors in schwann-like adult stem cells. J. Mol. Neurosci. 47, 619-630 (2012).

19. Faroni, A., Calabrese, F., Riva, M. A., Terenghi, G. \& Magnaghi, V. Baclofen modulates the expression and release of neurotrophins in Schwann-like adipose stem cells. J. Mol. Neurosci. 49, 233-243 (2013).

20. Piovesana, R., Melfi, S., Fiore, M., Magnaghi, V. \& Tata, A. M. M2 muscarinic receptor activation inhibits cell proliferation and migration of rat adiposemesenchymal stem cells. J. Cell Physiol. 233, 5348-5360 (2018).

21. El-Habta, R., Kingham, P. J. \& Backman, L. J. Adipose stem cells enhance myoblast proliferation via acetylcholine and extracellular signal-regulated kinase 1/2 signaling. Muscle Nerve. 57, 305-311 (2018).

22. Kingham, P. J., Kolar, M. K., Novikova, L. N., Novikov, L. N. \& Wiberg, M. Stimulating the neurotrophic and angiogenic properties of human adiposederived stem cells enhances nerve repair. Stem. Cells Dev. 23, 741-754 (2014).

23. de Luca, A. C., Faroni, A. \& Reid, A. J. Dorsal root ganglia neurons and differentiated adipose-derived stem cells: an in vitro co-culture model to study peripheral nerve regeneration. J. Vis. Exp. 96, 52543 (2015).

24. Brockes, J. P. \& Fields, K. L. R. M. Studies on cultured rat Schwann cells. I. Establishment of purified populations from cultures of peripheral nerve. Brain Res. 165, 105-118 (1979).

25. Bobis, S. \& Jarocha, D. M. M. Mesenchymal stem cells: characteristics and clinical applications. Folia. Histochem. Cytobiol. 44, 215-230 (1984).

26. Reid, A. J. et al. Nerve repair with adipose-derived stem cells protects dorsal root ganglia neurons from apoptosis. Neuroscience 199, 515-522 (2011).

27. Faroni, A., Smith, R. J. P., Lu, L. \& Reid, A. J. Human Schwann-like cells derived from adipose-derived mesenchymal stem cells rapidly de-differentiate in the absence of stimulating medium. Eur. J. Neurosci. 43, 417-430 (2016).

28. Krishnan, A. Neuregulin-1 Type I: A Hidden Power Within Schwann Cells for Triggering. Scie Signal. 6, 1-4 (2013).

29. Magnaghi V., Procacci P., Tata A. M. Chapter 15 Novel Pharmacological Approaches to Schwann Cells as Neuroprotective Agents for Peripheral Nerve 
Regeneration. 1st ed. Elsevier Inc., https://doi.org/10.1016/S0074-7742(09) 87015-3 (2009).

30. Hall, S. The response to injury in the peripheral nervous system. J. Bone Joint Surg. Br. 87, 1309-1319 (2005).

31. De Stefano, M. E., Toni, F., Orazi, V. D., Ortensi, A. \& Tata, A. M. Therapeutic approaches enhancing peripheral nerve regeneration. Adv. Biosci. Biotechnol. 04, 53-60 (2013).

32. Matera, C. \& Tata, A. Pharmacological approaches to targeting muscarinic acetylcholine receptors. Recent. Pat. CNS Drug Disco. 9, 85-100 (2014).

33. Di Bari, M. et al. Cytotoxic and genotoxic effects mediated by M2 muscarinic receptor activation in human glioblastoma cells. Neurochem. Int. 90, 261-270 (2015).

34. Gomez-Sanchez, J. A. et al. After nerve injury, lineage tracing shows that myelin and Remak Schwann cells elongate extensively and branch to form repair Schwann cells, which shorten radically on re-myelination. J. Neurosci. 37, 1453-17 (2017).

35. Birchmeier, C. \& Bennett, D. L. H. Neuregulin/ErbB signaling in developmental myelin formation and nerve repair. Curr. Top. Dev. Biol. 116, 45-64 (2016).

36. Fricker, F. R. \& Bennett, D. L. The role of neuregulin-1 in the response to nerve injury. Future Neurol. 6, 809-822 (2011).
37. Syed, N. \& Kim, H. A. Soluble neuregulin and Schwann cell myelination: a therapeutic potential for improving remyelination of adult axons. Mol. Cell Pharm. 2, 161-167 (2010).

38. Orbay, H., Little, C. J., Lankford, L., Olson, C. A. \& Sahar, D. E. The key components of Schwann cell-like differentiation medium and their effects on gene expression pattern of adipose-derived stem cells. Ann. Plast. Surg. 74, 584-588 (2015).

39. Mortimer A. E., Faroni A., Kilic M. A., Reid A. J. Maintenance of a Schwann-Like Phenotype In Differentiated Adipose-derived Stem Cells Requires The Synergistic Action Of Multiple Growth Factors. Stem. Cells Int. 2017, 1479137 (2017).

40. Parkinson, D. B. et al. c-Jun is a negative regulator of myelination. J. Cell Biol. 181, 625-637 (2008).

41. Wang, P. et al. miR-3075 Inhibited the Migration of Schwann Cells by Targeting Cntn2. Neurochem. Res. 43, 1879-1886 (2018).

42. Tang, J. M. et al. Acetylcholine Induces Mesenchymal Stem Cell Migration via Ca2+/PKC/ERK1/2 Signal Pathway. J. Cell Biochem. 113, 2704-2713 (2012).

43. Negro, S. et al. CXCL12a/SDF-1 from perisynaptic Schwann cells promotes regeneration of injured motor axon terminals. EMBO Mol. Med. 9, 1000-1010 (2017).

44. Liu, M. et al. Adipose-Derived Mesenchymal Stem Cells from the Elderly Exhibit Decreased Migration and Differentiation Abilities with Senescent Properties. Cell Transpl. 26, 1505-1519 (2017). 